\title{
Hydrogen-rich saline attenuates steroid-associated femoral head necrosis through inhibition of oxidative stress in a rabbit model
}

\author{
SHENG-LI HUANG, JIAN JIAO and HONG-WEI YAN \\ Department of Orthopedics, The Second Affiliated Hospital, School of Medicine, \\ Xi'an Jiaotong University, Xi'an, Shaanxi 710004, P.R. China \\ Received September 16, 2014; Accepted July 31, 2015
}

DOI: $10.3892 /$ etm.2015.2883

\begin{abstract}
A growing body of evidence suggests that hydrogen is a novel, selective antioxidant that exerts a protective effect against organ damage. The present study investigated the effect of hydrogen-rich saline on corticosteroid-induced necrosis of the femoral head in an animal model established using prednisolone. A total of 30 healthy, male, adult New Zealand white rabbits were randomly divided into two groups: Hydrogen-rich saline (treated with hydrogen-rich saline via intraperitoneal injection) and placebo (treated with normal saline). At the set time-points, the structure of the femoral head was examined using a microscope; the concentrations of glutathione (GSH), lipid peroxide (LPO), vascular endothelial growth factor (VEGF) and thrombomodulin (TM) in the plasma were measured and the microvessel density was quantified. The results showed that hydrogen-rich saline significantly decreased the levels of VEGF, TM and LPO and increased the GSH level in steroid-associated necrosis of the femoral head in the rabbit model. A significant increase in the microvessel density was observed in the hydrogen-rich saline group. Histopathological staining confirmed the results of the biochemical analysis. The present study demonstrates that hydrogen treatment may alleviate steroid-associated osteonecrosis by inhibiting oxidative stress. Hydrogen-rich saline may provide an alternative treatment for steroid-associated necrosis of the femoral head.
\end{abstract}

\section{Introduction}

Steroids are useful drugs that can be applied in numerous serious diseases, since they are capable of profoundly

Correspondence to: Professor Hong-Wei Yan, Department of Orthopedics, The Second Affiliated Hospital, School of Medicine, Xi'an Jiaotong University, 157 Xiwulu, Xi'an, Shaanxi 710004, P.R. China

E-mail: hongweiyey@sina.com

Key words: femoral head necrosis, hydrogen, oxidative stress, rabbit affecting the disease course; however, particularly with high dosages and prolonged use, the incidence of steroid-related complications and side effects is high, and this can lead to serious consequences, such as avascular necrosis of the bone. Steroid-associated osteonecrosis, in which the femoral head is most often affected, was first recognized in 1957 (1). Femoral head necrosis occurs primarily in young and middle-aged individuals and has a high disability rate. The condition induces partial or complete loss of the ability to walk, thus seriously affecting the patient's quality of life (2). Since femoral head necrosis is a progressive, pathological process, the femoral head will deform or even collapse without treatment (3). Early treatment is therefore crucial for preserving the femoral head, and there is an urgent requirement for the development of novel therapeutic strategies.

A number of studies have reported promising results regarding the role of hydrogen in treating diseases in the brain $(4,5)$, heart (6), liver (7), kidney (8), intestine (9), lung (10) and spinal cord (11). Hydrogen has a positive effect in inhibiting oxidative stress. Hydrogen gas is flammable, explosive and difficult to store and use; however, hydrogen gas-saturated saline, also known as hydrogen-rich saline, is safe, economical and easily available, and is universally employed in medicine $(12,13)$. Injecting hydrogen-rich saline into the intraperitoneal cavity is an easy and effective method that can be readily adapted for potential clinical practice (14).

Hydrogen-rich saline may be a promising, safe and effective agent for the treatment of a variety of diseases; however, to the best of our knowledge, there are no studies on its role in preventing or treating femoral head necrosis. Given the fact that hydrogen-rich saline has highly protective properties, we hypothesized that the application of the agent would exert a therapeutic effect on femoral head necrosis. In the present study, the role of hydrogen-rich saline in an animal model of femoral head necrosis, as well as the possible mechanism underlying its effect, was investigated.

\section{Materials and methods}

Animals. The present study was approved by the Animal Experiment Committee of Xi'an Jiaotong University (Xi'an, 
China). The principles of laboratory animal care were followed, all procedures were conducted in accordance with the guidelines established by the National Institutes of Health and every effort was made to minimize the suffering of the animals. A total of 30 healthy, adult, male New Zealand white rabbits weighing $2.0-2.5 \mathrm{~kg}$ (mean, $2.25 \pm 0.15 \mathrm{~kg}$ ) were supplied by the Center of Experimental Animals of Xi'an Jiaotong University School of Medicine. All rabbits were housed in standard cages with food and water ad libitum under a natural day/night cycle.

Hydrogen-rich saline and other reagents. Hydrogen was dissolved in physiological saline for $6 \mathrm{~h}$ under a pressure of 0.4 MPa to a supersaturated level. The obtained hydrogen-rich saline was sterilized by $\gamma$-radiation and stored under atmospheric pressure at $4^{\circ} \mathrm{C}$ in an aluminum bag with no dead volume. The saline was freshly prepared every week to ensure a concentration of $>0.6 \mathrm{mmol} / \mathrm{l}$. Gas chromatography was used to confirm the content of hydrogen in the saline (5).

Rabbit thrombomodulin (TM) and vascular endothelial growth factor (VEGF) ELISA kits were purchased from Cusabio Biotech Co., Ltd. (Wuhan, China). Rabbit glutathione (GSH) and rabbit lipid peroxide (LPO) ELISA kits were obtained from Shanghai Bohua Biological Technology Co., Ltd. (Shanghai, China). Anti-CD34 polyclonal antibody (bs-0646R) was purchased from Boosen Biological Technology Co., Ltd. (Beijing, China). A 3,3'-diaminobenzidine (DAB) staining kit was obtained from Sequoia Jinqiao Biological Technology Co., Ltd (Beijing, China). Lipopolysaccharide (LPS) and prednisolone were purchased from Sigma-Aldrich (St Louis, MO, USA).

Animal model. The animal model of steroid-associated osteonecrosis was prepared as previously described $(15,16)$. Briefly, after 1 week of acclimation, all rabbits received one intravenous injection of LPS $(10 \mu \mathrm{g} / \mathrm{kg})$. After $24 \mathrm{~h}$, an intragluteal injection of prednisolone was performed at a dosage of $20 \mathrm{mg} / \mathrm{kg}$, once per day, for 3 days.

Hydrogen-rich saline. The animals were randomly divided into two groups ( $\mathrm{n}=15$ per group). Compared with the intravenous and local injections of hydrogen-rich saline, the intraperitoneal injection is more convenient for long-term treatment and was therefore selected in the present study. Rabbits in the hydrogen-rich saline group were treated with an intraperitoneal injection of hydrogen-rich saline at a dosage of $5 \mathrm{mg} / \mathrm{kg} / \mathrm{day}$, once per day, for 14 consecutive days, while those in the placebo group received normal saline.

Biochemical analysis. Tests for GSH and LPO were performed immediately prior to the LPS injection and 3, 5, 7 and 14 days after saline treatment. At these time-points, blood samples ( $2 \mathrm{ml}$ in each group) were collected from the auricular arteries of the rabbits under anesthesia, and plasma was obtained by transferring the blood sample into a tube containing 3.8\% sodium citrate anticoagulant $(0.2 \mathrm{ml})$ for centrifugation at $1,500 \mathrm{x} \mathrm{g}$ for $10 \mathrm{~min}$ at $4^{\circ} \mathrm{C}$. The plasma was then stored at $-70^{\circ} \mathrm{C}$ for the quantitative determination of GSH and LPO levels using ELISA. Measurements of VEGF and TM were performed prior to model establishment, as well as 2, 4 and 6 weeks after saline treatment. Plasma samples were obtained and used for the quantitative determination of VEGF and TM using the aforementioned method.

Histopathology and immunohistochemistry. At 2, 4 and 6 weeks after saline treatment, 5 randomly selected rabbits from each group were sacrificed by an intravenous injection of air and the femoral heads were harvested. The femoral heads were fixed in neutral formaldehyde, decalcified, dehydrated with an alcohol series and embedded in paraffin. The samples were the cut into 5- $\mu \mathrm{m}$ sections along the coronal plane for the subsequent pathological staining using hematoxylin and eosin (H\&E) and immunohistochemistry.

In order to highlight the microvessels, the sections were stained by immunohistochemistry for the detection of the angiogenic marker CD34 in endothelial cells. The deparaffinized and hydrated sections were washed with phosphate buffer solution three times, for $5 \mathrm{~min}$ each time, and were then treated with $3 \%$ hydrogen peroxide for 20 min to block endogenous peroxidase activity. The slides were immersed in $0.01 \mathrm{~mol} / \mathrm{l}$ citrate buffer $(\mathrm{pH} 6.0$ ), heated in a microwave at a constant temperature of $92-98^{\circ} \mathrm{C}$ for $10 \mathrm{~min}$ and then rinsed with phosphate buffer solution three times. After $1 \mathrm{~h}$ of incubation with $10 \%$ goat serum, the primary antibody was added $(1: 100)$ and the sections were incubated at room temperature for another hour, followed by overnight incubation at $4^{\circ} \mathrm{C}$. Following three washes in phosphate buffer solution, the sections were incubated with biotinylated goat anti-rabbit IgG secondary antibody (1:200; bs-0295G-Bio; Boosen Biological Technology Co., Ltd., Beijing, China) for $2 \mathrm{~h}$ at room temperature, rinsed and placed in avidin-peroxidase conjugate solution for $2 \mathrm{~h}$. The horseradish peroxidase chromogenic substrate $0.05 \%$ DAB was added for visualization. The sections were then counterstained with hematoxylin, dehydrated and mounted. Appropriate sections were selected to be used as positive and negative controls.

Microvessel counting was performed using the method previously reported by Weidner et al $(17,18)$. Briefly, areas of the most intense CD34 antigen staining were identified by light microscopy at low power. The counting of the microvessels per field was performed using a higher-power microscope (magnification, x200) manually from 5 random fields. The results are expressed as the average number of microvessels identified within the 5 fields. Any endothelial cell or cell cluster positive for CD34 antigen and clearly separate from an adjacent cluster was considered a single, countable microvessel.

Statistical analysis. All statistical analyses were performed using SPSS 16.0 software (SPSS Inc., Chicago, IL, USA). Data are expressed as the mean \pm standard deviation. The comparison between the placebo and hydrogen-rich saline groups was performed using the Student's $t$-test. A one-way analysis of variance was used to compare matched data at multiple time-points. A two-tailed $\mathrm{P}<0.05$ was considered to indicate a statistically significant difference.

\section{Results}

General data. There were no mortalities at any time during the experiment, and no side effects of the hydrogen-rich saline on the overall well-being of the rabbits were observed in either group. 

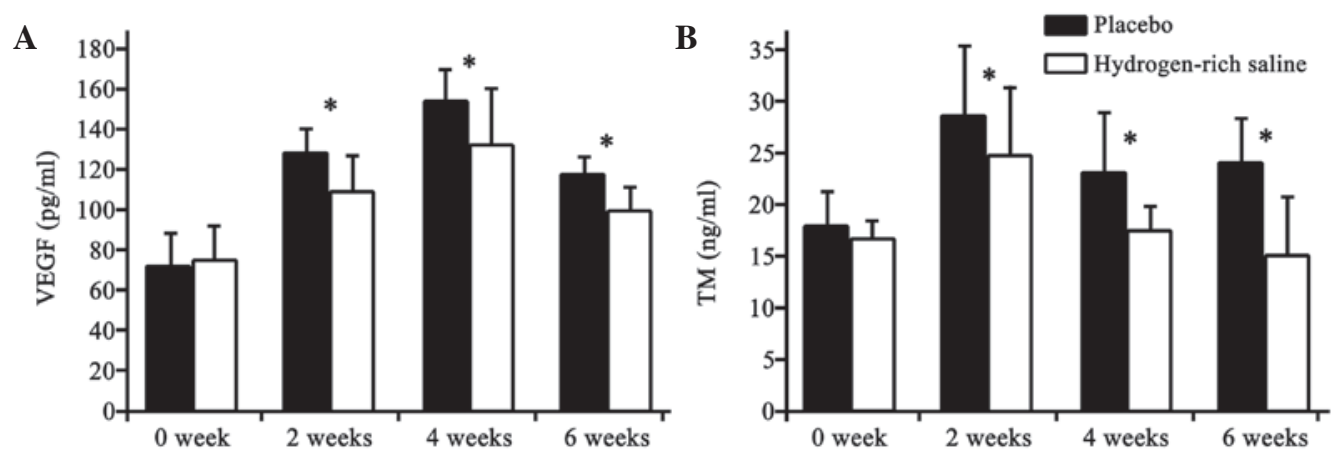

Figure 1. VEGF and TM plasma levels of rabbits at different time-points in the placebo and hydrogen-rich saline groups (n=15 per group). Plasma levels of (A) VEGF and (B) TM immediately prior to lipopolysaccharide injection and at 2, 4 and 6 weeks after saline treatment. " $\mathrm{P}<0.05$, hydrogen-rich saline group vs. placebo group. VEGF, vascular endothelial growth factor; TM, thrombomodulin.
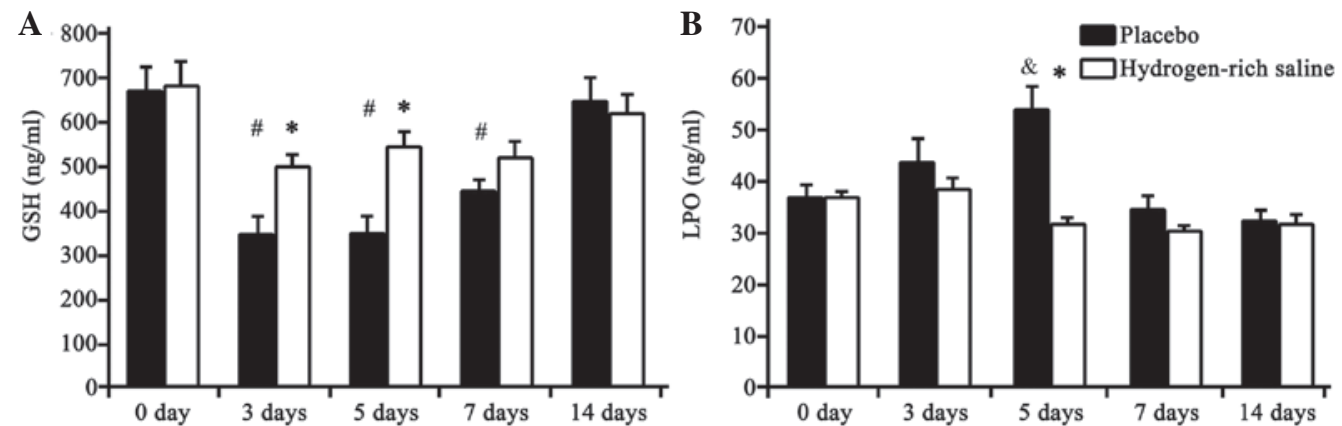

Figure 2. GSH and LPO plasma levels at different time-points in the placebo and hydrogen-rich saline groups ( $\mathrm{n}=15$ per group). Plasma levels of (A) GSH and (B) LPO immediately prior to lipopolysaccharide injection and at 3,5,7 and 14 days after saline treatment. "P<0.05, hydrogen-rich saline group vs. placebo group; ${ }^{~} \mathrm{P}<0.05$, both groups vs. baseline; ${ }^{\circledR} \mathrm{P}<0.05$, placebo group vs. baseline. $\mathrm{GSH}$, glutathione; $\mathrm{LPO}$, lipid peroxide.

Plasma levels of VEGF, TM, GSH and LPO. The concentrations of VEGF and TM in the plasma decreased significantly following the hydrogen-rich saline treatment (Fig. 1). The differences between the hydrogen-rich saline and placebo groups were significant $(\mathrm{P}<0.05)$.

The activity of GSH and LPO was determined as an indicator of oxidative stress. As shown in Fig. 2, the GSH concentration in the plasma was significantly increased in the hydrogen-rich saline group at days 3 and 5 compared with that in the placebo group, and the LPO concentration was significantly decreased at day 5 . No significant differences between the groups were observed on days 7 and 14 .

Compared with baseline levels (immediately prior to the LPS injection), significant differences were observed in the GSH level in both the placebo and hydrogen-rich saline groups between days 3 and 7 . With regard to LPO, a significant increase was only identified on day 5 in the placebo group. These differences gradually diminished with the passage of time.

Histopathological observations. The degree of osteonecrosis in the femoral head was assessed using H\&E staining. The trabecular bone, lacunae, bone marrow and fat tissue were visualized using light microscopy (Fig. 3). Histological examination demonstrated that the condition of the femoral head in the placebo group became gradually aggravated as the time passed. A diffuse presence of empty lacunae or pyknotic nuclei of osteocytes was observed in the trabeculae at week 2, and thin trabeculae with a disordered texture and hypertrophic fat cells were noted. At week 4 , thinner bone trabeculae were observed, with breakage of a part of the bone trabeculae. A number of lacunae were empty, and abundant adipose cells were found in the marrow cavity. At week 6, there was sparser trabecular bone with empty lacunae compared with week 4 ; however, the empty bone lacunae had clearly increased. The bone marrow had been partly replaced by fat tissue. There was no clear indication of new bone formation.

Histological examination revealed improvement in the trabecular bone, lacunae, bone marrow and fat tissue following treatment with hydrogen-rich saline. In the hydrogen-rich saline group, the bone trabeculae were thinner and no diffuse, empty lacunae were observed. The number of adipose cells had almost returned to normal at week 2 . Some cells exhibited vacuolization or pyknosis at week 4 , and empty bone lacunae were occasionally found. At the end of the experiment (week 6) a number of empty lacunae and osteocytes with pyknotic nuclei were found within the trabeculae. There were no signs of an inflammatory response (inflammatory cells).

Microvascular characteristics. Microvascular density was evaluated in order to ascertain vascular changes. All microvessels were highlighted using immunohistochemical staining. The results revealed a significant increase in the microvascular density in the hydrogen-rich saline group compared with that in the placebo group (Fig. 4) $(\mathrm{P}<0.05)$. 

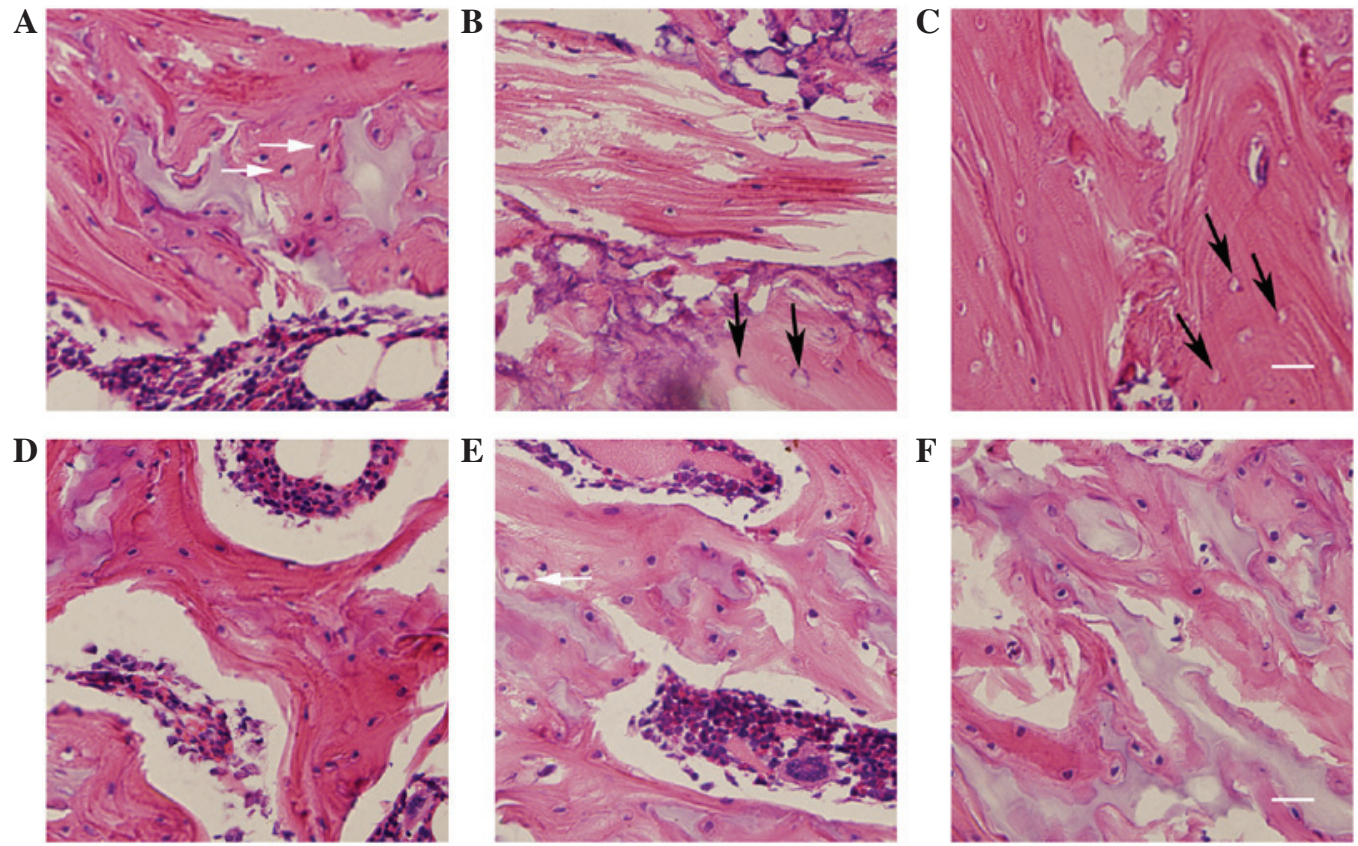

Figure 3. Histological changes in the trabecular bone microstructure in the femoral head. Histological changes at weeks 2, 4 and 6 in the (A-C) placebo group and (D-F) hydrogen-rich saline group, respectively. The histopathology of the hydrogen-rich saline group showed a significant improvement in the trabecular bone microstructure compared with the placebo group. White arrows indicate pyknotic nuclei of osteocytes; black arrows indicate empty lacunae. Scale bar $=100 \mu \mathrm{m}$.

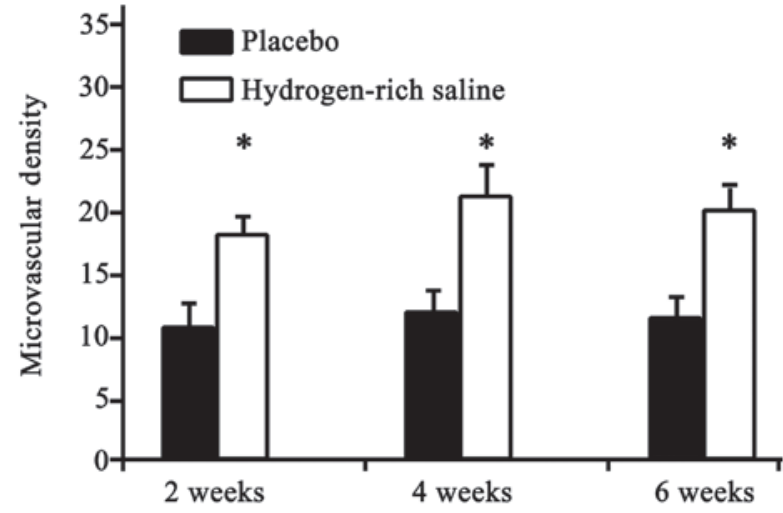

Figure 4. Microvascular density at 2, 4 and 6 weeks after saline treatment in the placebo and hydrogen-rich saline groups ( $n=15$ per group). Microvascular density in the hydrogen-rich saline group was significantly increased for $\geq 6$ weeks compared with that in the placebo group. ${ }^{*} \mathrm{P}<0.05$, hydrogen-rich saline group vs. placebo group.

\section{Discussion}

Accumulating evidence suggests that hydrogen has medicinal properties (4-11,19); however, the molecular mechanism has yet to be elucidated. Little is known about the potential role of hydrogen in femoral head necrosis. In the present study, the effect of hydrogen-rich saline on femoral head necrosis was evaluated using histopathology, immunohistochemistry and the quantitative determination of the plasma levels of VEGF, TM, GSH and LPO in an animal model, which was established using prednisolone. The levels of the biochemical indicators VEGF and TM were decreased in the plasma samples treated with hydrogen-rich saline, suggesting that the treatment relieved the microvascular injury. The microvascular and histopathological results were similar, indicating a therapeutic effect of hydrogen-rich saline on femoral head necrosis. To the best of our knowledge, the present study is the first to demonstrate a protective role of hydrogen-rich saline in femoral head necrosis. Furthermore, it was found that hydrogen-rich saline could markedly decrease oxidative stress.

Femoral head necrosis is known as Chandler's disease in adults and Legg-Calve-Perthes disease in children (20). Osteonecrosis indicates cellular death in both bone and marrow tissue. The pathogenesis of this condition is extremely complex and still not completely understood, and there is currently no satisfactory clinical treatment available. Animal models of corticosteroid-induced bone necrosis have been widely used in the investigation of the etiology and therapeutics of femoral head necrosis (21-23). In the present study, the animal models were established in adult male New Zealand white rabbits using prednisolone. Only male rabbits were selected in order to avoid gender variation in the incidence of osteonecrosis. The high degree of osteonecrosis revealed by the histological examination in the placebo group indicated successful modeling.

VEGF and TM levels in the plasma are indexes of endothelial injury $(24,25)$. In the present experiment, the plasma level of VEGF increased in the first 4 weeks and then decreased thereafter. This indicates that early necrosis of the femoral head promotes VEGF secretion. The TM level peaked at week 2 and began to fall at week 4 . The changes in VEGF lagged behind TM. The treatment with hydrogen-rich saline significantly decreased the levels of both VEGF and TM. These results indicate that hydrogen is able to prevent endothelial injury, as shown by the fact that the significantly increased VEGF and TM levels in the placebo group were attenuated 
in the hydrogen-rich saline group. We therefore suggest that hydrogen-rich saline ameliorates femoral head necrosis by inhibiting the VEGF and TM expression. The determination of microvessel density in the present study provided additional information and showed that the hydrogen-rich saline treatment resulted in the significant improvement in the microvascular condition. Hydrogen-rich saline therefore prevents the decrease in microvascular density. The protective effect of hydrogen-rich saline against femoral head necrosis was further confirmed histologically. The significant decrease in VEGF and TM concentrations and the marked increase in microvascular density following hydrogen-rich saline treatment indicate that hydrogen-rich saline could prevent the development of osteonecrosis.

Since the hydrogen molecule is electrically neutral and considerably smaller than other antioxidants, it can easily penetrate membranes and enter organelles $(12,26)$; however, it remains unclear exactly what type of oxidative stress is involved in femoral head necrosis. GSH stabilizes the lysosomal membranes to suppress injury to the vascular endothelium. LPO is a biochemical indicator of tissue injury and is closely associated with vascular injury (27). GSH and LPO in the plasma are biochemical markers indicating the level of oxidative stress, and GSH has often been used in the study of osteonecrosis, due to the fact that GSH levels and oxidative stress are associated with osteonecrosis (28-30). In the present experiment, hydrogen-rich saline treatment significantly increased the GSH levels in the plasma, while it decreased the LPO levels, indicating that hydrogen-rich saline has a protective effect against oxidative stress in femoral head necrosis. The results therefore showed that hydrogen-rich saline could suppress activated oxidative stress in endothelial injury at an early stage during the development of femoral head necrosis.

In conclusion, the results of this study validated the therapeutic potential of hydrogen-rich saline by demonstrating that an injection of this agent can alleviate steroid-associated osteonecrosis in a rabbit model. The present results also indicate that hydrogen can protect the endothelium from oxidative stress. The suppression of the activated oxidative stress leading to intravascular endothelium injury could be involved in the underlying mechanism; however, the exact mechanism of the therapeutic role of hydrogen-rich saline in femoral head necrosis requires further research.

\section{References}

1. Pietrogrande V and Mastomarino R: Osteopathy from prolonged cortisone treatment. Ortop Traumatol Apparate Motore 25: 791-810, 1957 (In Italian).

2. Zhang YG, Wang X, Yang Z, Zhang H, Liu M, Qiu Y and Guo X: The therapeutic effect of negative pressure in treating femoral head necrosis in rabbits. PLoS One 8: e55745, 2013.

3. Wang C, Peng J and Lu S: Summary of the various treatments for osteonecrosis of the femoral head by mechanism: A review. Exp Ther Med 8: 700-706, 2014.

4. Ji Q, Hui K, Zhang L, Sun X, Li W and Duan M: The effect of hydrogen-rich saline on the brain of rats with transient ischemia. J Surg Res 168: e95-e101, 2011

5. Ohsawa I, Ishikawa M, Takahashi K, Watanabe M, Nishimaki K, Yamagata K, Katsura K, Katayama Y, Asoh S and Ohta S: Hydrogen acts as a therapeutic antioxidant by selectively reducing cytotoxic oxygen radicals. Nat Med 13: 688-694, 2007.
6. Hayashida K, Sano M, Ohsawa I, Shinmura K, Tamaki K, Kimura K, Endo J, Katayama T, Kawamura A, Kohsaka S, et al: Inhalation of hydrogen gas reduces infarct size in the rat model of myocardial ischemia-reperfusion injury. Biochem Biophys Res Commun 373: 30-35, 2008.

7. Fukuda K, Asoh S, Ishikawa M, Yamamoto Y, Ohsawa I and Ohta S: Inhalation of hydrogen gas suppresses hepatic injury caused by ischemia/reperfusion through reducing oxidative stress. Biochem Biophys Res Commun 361: 670-674, 2007.

8. Wang F, Yu G, Liu SY, Li JB, Wang JF, Bo LL, Qian LR, Sun XJ and Deng XM: Hydrogen-rich saline protects against renal ischemia/reperfusion injury in rats. J Surg Res 167: e339-e344, 2011.

9. Buchholz BM, Kaczorowski DJ, Sugimoto R, Yang R, Wang Y Billiar TR, McCurry KR, Bauer AJ and Nakao A: Hydrogen inhalation ameliorates oxidative stress in transplantation induced intestinal graft injury. Am J Transplant 8: 2015-2024, 2008.

10. Kawamura T, Huang CS, Tochigi N, Lee S, Shigemura N, Billiar TR, Okumura M, Nakao A and Toyoda Y: Inhaled hydrogen gas therapy for prevention of lung transplant-induced ischemia/reperfusion injury in rats. Transplantation 90: 1344-1351, 2010.

11. Huang Y, Xie K, Li J, Xu N, Gong G, Wang G, Yu Y, Dong H and Xiong L: Beneficial effects of hydrogen gas against spinal cord ischemia-reperfusion injury in rabbits. Brain Res 1378: 125-136, 2011.

12. Li S, Lu D, Zhang Y and Zhang Y: Long-term treatment of hydrogen-rich saline abates testicular oxidative stress induced by nicotine in mice. J Assist Reprod Genet 31: 109-114, 2014.

13. Du Z, Jia H, Liu J, Zhao X, Wang Y and Sun X: Protective effects of hydrogen-rich saline in uncontrolled hemorrhagic shock. Exp Ther Med 7: 1253-1258, 2014.

14. Zheng $\mathrm{H}$ and $\mathrm{Yu}$ YS: Chronic hydrogen-rich saline treatment attenuates vascular dysfunction in spontaneous hypertensive rats. Biochem Pharmacol 83: 1269-1277, 2012.

15. Zhang G, Qin L, Sheng H, Wang XL, Wang YX, Yeung DK, Griffith JF, Yao XS, Xie XH, Li ZR, et al: A novel semisynthesized small molecule icaritin reduces incidence of steroid-associated osteonecrosis with inhibition of both thrombosis and lipid-deposition in a dose-dependent manner. Bone 44: 345-356, 2009

16. Qin L, Zhang G, Sheng H, Yeung KW, Yeung HY, Chan CW, Cheung WH, Griffith J, Chiu KH and Leung KS: Multiple bioimaging modalities in evaluation of an experimental osteonecrosis induced by a combination of lipopolysaccharide and methylprednisolone. Bone 39: 863-871, 2006.

17. Weidner N, Folkman J, Pozza F, Bevilacqua P, Allred EN, Moore DH, Meli S and Gasparini G: Tumor angiogenesis: A new significant and independent prognostic indicator in early-stage breast carcinoma. J Natl Cancer Inst 84: 1875-1887, 1992.

18. Weidner N, Semple JP, Welch WR and Folkman J: Tumor angiogenesis and metastasis-correlation in invasive breast carcinoma. N Engl J Med 324: 1-8, 1991.

19. Hanaoka T, Kamimura N, Yokota T, Takai S and Ohta S: Molecular hydrogen protects chondrocytes from oxidative stress and indirectly alters gene expressions through reducing peroxynitrite derived from nitric oxide. Med Gas Res 1: 18, 2011.

20. McLennan MK and Margolis M: Radiology rounds. Inflammatory bowel disease (ulcerative colitis) with steroid-induced avascular necrosis of the femoral heads. Can Fam Physician 40: 1272-1274, 1994.

21. Guan XY and Han D: Role of hypercoagulability in steroid-induced femoral head necrosis in rabbits. J Orthop Sci 15: 365-370, 2010.

22. He W, Xu C, Fan Y, Fang B, Li X, Wang H, Liu S and Yuan H: Effects of the Chinese drugs for activating blood circulation on plasma TXB2 and 6-keto-PGF1alpha contents in rabbits with glucocorticoid-induced femoral head necrosis. J Tradit Chin Med 24: 233-237, 2004.

23. ZhuH,CaiX,LinT,ShiZand Yan S:Low-intensity pulsedultrasound enhances bone repair in a rabbit model of steroid-associated osteonecrosis. Clin Orthop Relat Res 473: 1830-1839, 2015.

24. Zhang C, Li Y, Cornelia R, Swisher S and Kim H: Regulation of VEGF expression by HIF-1 $\alpha$ in the femoral head cartilage following ischemia osteonecrosis. Sci Rep 2: 650, 2012.

25. Aksoy MC1, Aksoy DY, Haznedaroglu IC, Sayinalp N, Kirazli S and Alpaslan M: Thrombomodulin and GFC levels in Legg-Calve-Perthes disease. Hematology 13: 324-328, 2008.

26. Zhang CB, Tang YC, Xu XJ, Guo SX and Wang HZ: Hydrogen gas inhalation protects against liver ischemia/reperfusion injury by activating the NF- $\kappa \mathrm{B}$ signaling pathway. Exp Ther Med 9: 2114-2120, 2015 
27. Uzel N, Sivas A, Uysal M and Oz H: Erythrocyte lipid peroxidation and glutathione peroxidase activities in patients with diabetes mellitus. Horm Metab Res 19: 89-90, 1987.

28. Ichiseki T, Kaneuji A, Ueda Y, Nakagawa S, Mikami T, Fukui K and Matsumoto T: Osteonecrosis development in a novel rat model characterized by a single application of oxidative stress. Arthritis Rheum 63: 2138-2141, 2011.
29. Ichiseki T, Kaneuji A, Katsuda S, Ueda Y, Sugimori T and Matsumoto T: DNA oxidation injury in bone early after steroid administration is involved in the pathogenesis of steroid-induced osteonecrosis. Rheumatology (Oxford) 44: 456-460, 2005.

30. Ichiseki T, Matsumoto T, Nishino M, Kaneuji A and Katsuda S: Oxidative stress and vascular permeability in steroid-induced osteonecrosis model. J Orthop Sci 9: 509-515, 2004. 